\title{
Mode-matching Generalised Scattering Matrix Based Electromagnetics Performance Analysis of Thick Frequency Selective Surfaces for Airborne Applications
}

\author{
Shiv Narayan*, R.U. Nair, and R.M. Jha \\ CSIR-National Aerospace Laboratories, Bangalore-560 017, India \\ *E-mail: shivnarayan@nal.res.in
}

\begin{abstract}
Electromagnetic (EM) performance of thick aperture type frequency selective surfaces (FSS) has been analyzed using mode-matching generalized scattering matrix (MM-GSM) technique for airborne radome applications. The MM-GSM technique facilitates accurate EM performance predictions for both single and multi-layered FSS structures as it includes evanescent and propagating modes in the EM analysis. The computed transmission efficiency of the thick FSS structure over X-band at normal and near normal incidence shows excellent matching with the reported results in open domain. he study has been extended to determine the FSS band-pass transmission characteristics for incidence angles as high as $80^{\circ}$ encountered in highly-streamlined nosecone radomes. Further, the dependence of transmission efficiency of the FSS structure on the dielectric parameters of the material filled within the apertures has also been analyzed. The present work shows that the proposed thick aperture-type FSS structure has potential applications in the design of airborne radomes.
\end{abstract}

Keywords: Frequency selective surface, generalized scattering matrix, mode-matching technique, thick FSS

\section{NOMENCLATURE}

\begin{tabular}{ll}
$\alpha$ & Skew angle \\
$\beta_{p q}$ & Propagation constant of waveguide mode \\
$\gamma_{m n}$ & Propagation constant of floquet mode \\
$\varepsilon_{r}$ & Dielectric constant \\
$\eta_{0}$ & Free-space impedance \\
$\rho$ & Position vector of unit cell \\
$\tau_{m n}$ & Transverse wave number of floquet mode \\
$a_{m}^{ \pm}$ & Amplitude of floquet mode \\
$A^{ \pm}$ & Floquet mode coefficient in region-1 \\
$b_{p}^{ \pm}$ & Amplitude of waveguide mode \\
$B^{ \pm}$ & Waveguide mode coefficient in region-2 \\
$C^{ \pm}$ & Coupling power integral \\
$E_{T}^{f}$ & Floquet mode coefficient in region-3 \\
$E_{T}^{w}$ & Transverse component of electric field for \\
$H_{T}^{f}$ & Floquet mode \\
$H_{T}^{w}$ & Transverse component of electric field for \\
& Waveguide mode \\
$k_{p q}$ & Transverse component of magnetic field for \\
$m, n$ & Floquet mode \\
$p, q$ & Transverse component of magnetic field for \\
$Q$ & waveguide mode \\
$R$ & Transverse wave number of waveguide \\
$R$ & mode \\
\hline$R$ & Number of Floquet mode \\
& Number of waveguide mode \\
& Power integral for waveguide (aperture) \\
region & Power integral for free-space region
\end{tabular}

\section{INTRODUCTION}

In aerospace applications, frequency selective surfaces (FSS) are widely used in the design of airborne radomes and radar absorbing structures (RAS). Generally thick aperture-type FSS are used to enhance the strength and hardness of the radome structures ${ }^{1}$, to improve the band-pass filter characteristics, or to avoid radiation from microwave sources ${ }^{2}$. Accurate evaluation of EM performance of such FSS structure is essential for these applications. In view of this, number of numerical methods for analyzing FSS structures have been reported in the open literature namely; equivalent circuit analysis (ECA) method of moment (MoM) $)^{4}$, finite element method (FEM) ${ }^{5}$, and transmission line method ${ }^{6}$ etc. The ECA is best suited for thin FSS structures, but not for multilayered structures. MoM fails to analyse an infinite array structure where the number of basis functions is equal to the number of expansion modes ${ }^{4}$. In case of FEM, the complexity in computation increases for standard problems with the implementation of absorbing boundary conditions. Further the accuracy of FEM method reduces at high incidence angles. Transmission line method is also less accurate at oblique incidence angles. Compared to these methods, mode-matching-generalized scattering matrix (MM-GSM) method is more efficient and accurate for both single and multi-layered FSS structures at normal as well as oblique incidence as this method includes both evanescent and propagating modes in the analysis. Further, matching of the total mode fields at each junction between uniform sections of the structure enhances the accuracy of computation.

Since the thick FSS has better band-pass stability, high

Received 5 June 2012, revised 17 April 2013, online published 16 May 2013 
transmission efficiency inside the band, extremely good outof-band attenuation, and high structural rigidity, as compared to thin FSS, it is generally preferred for the airborne radome applications. Hence in this paper, the EM performance analysis of thick rectangular aperture-type FSS has been carried out using MM-GSM technique for its effective usage in the design of airborne radome.

\section{THEORETICAL ASPECTS}

Consider a thick perfectly conducting screen, perforated by an array of rectangular apertures lie in XY-plane, as shown in Fig. 1. The screen is illuminated by EM plane wave at arbitrary incidence angle $\theta$, lying on the YZ-plane. The FSS structure has a periodicity in the X-and Y-directions. Here $\alpha$ is the skew angle, i.e., the angle between the directions of periodicities. The fields on both side of the screen are expanded in terms of floquet modes with a propagation constant $\gamma_{m n}$ along the $z$-axis as $^{7}$

$$
\begin{gathered}
E_{T}^{f}(\rho, z)=\sum_{m}\left(a_{m}^{+}+a_{m}^{-}\right) E_{T_{m}}^{f}(\rho) \\
H_{T}^{f}(\rho, z)=\sum_{m}\left(a_{m}^{+}-a_{m}^{-}\right) H_{T_{m}}^{f}(\rho)
\end{gathered}
$$

represents the number of waveguide modes inside the aperture.

By matching the transverse components of electric and magnetic fields at the aperture $\mathrm{z}=0$, a system of linear equations for waveguide coefficients are obtained ${ }^{7}$ as

$$
\begin{aligned}
& \sum_{m}\left(A_{m}^{ \pm}+A_{m}^{-}\right) R_{m n}=\sum_{p}\left(B_{p}^{+}+B_{p}^{-}\right) C_{p n} \\
& \sum_{m}^{\text {and }}\left(A_{m}^{ \pm}-A_{m}^{-}\right) C_{n p}^{*}=\sum_{p}\left(B_{p}^{+}-B_{p}^{-}\right) Q_{p q}
\end{aligned}
$$

where $A_{m}^{ \pm}$and $B_{p}^{ \pm}$represent the mode coefficients for floquet and waveguide modes respectively. Here $R$ and $Q$ are the power integrals for the free-space and aperture region respectively. $C$ represents the coupling power integrals between the free-space and aperture modes.

\subsection{Scattering Matrix Formulation}

To render the scattering matrix of the proposed FSS structure, the scattering matrix at the interfaces $z=0$, and $z=h$ has been evaluated using MM-GSM technique and then cascaded to obtain the scattering matrix of the whole FSS structure. The side-view of the FSS is shown in Fig. 2, where $\mathrm{A}, \mathrm{B}$, and $\mathrm{C}$ are the mode coefficients in the region-1, 2, and
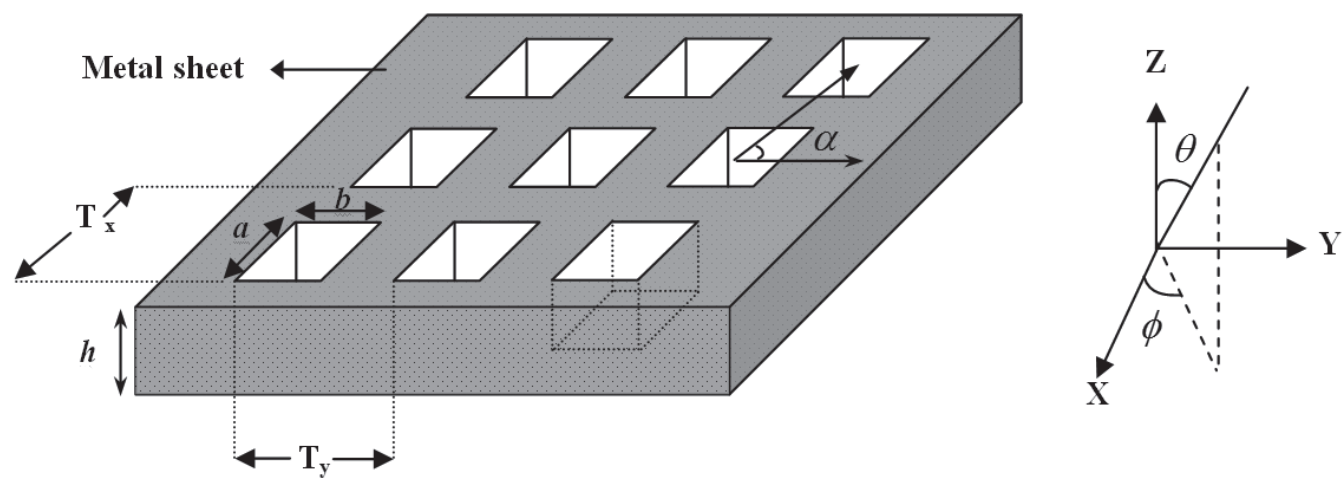

Figure 1. Schematic of a thick FSS perforated by rectangular aperture.

where $a_{m}^{ \pm}(z)=A_{m}^{ \pm} e^{ \pm \gamma_{m n^{z}}}$ represent the amplitudes of Floquet modes, and $\rho=x \hat{x}+y \hat{y}$ is the position vector of unit cell. Here $E_{T_{m}}^{f}$ and $H_{T_{m}}^{f}$ are the transverse components of electric and magnetic fields for floquet modes respectively. Subscript $m$ represents the number of floquet modes outside the structure.

The fields inside the aperture region are expressed in terms of the classical waveguide modes with propagation constant $\beta_{p q}$ as

$$
\begin{aligned}
& E_{T}^{w}(\rho, z)=\sum_{p}\left(b_{p}^{+}+b_{p}^{-}\right) E_{T_{p}}^{w}(\rho) \\
& H_{T}^{w}(\rho, z)=\sum_{p}\left(b_{p}^{+}-b_{p}^{-}\right) H_{T_{p}}^{w}(\rho)
\end{aligned}
$$

where $E_{T p}^{w}$ and $H_{T_{p}}^{w}$ represent the transverse components of electric and magnetic fields respectively. Here $b_{p}^{ \pm}(z)=B_{p}^{ \pm} e^{ \pm \beta_{p q}}$ represent the amplitudes of waveguide modes. Subscript $p$

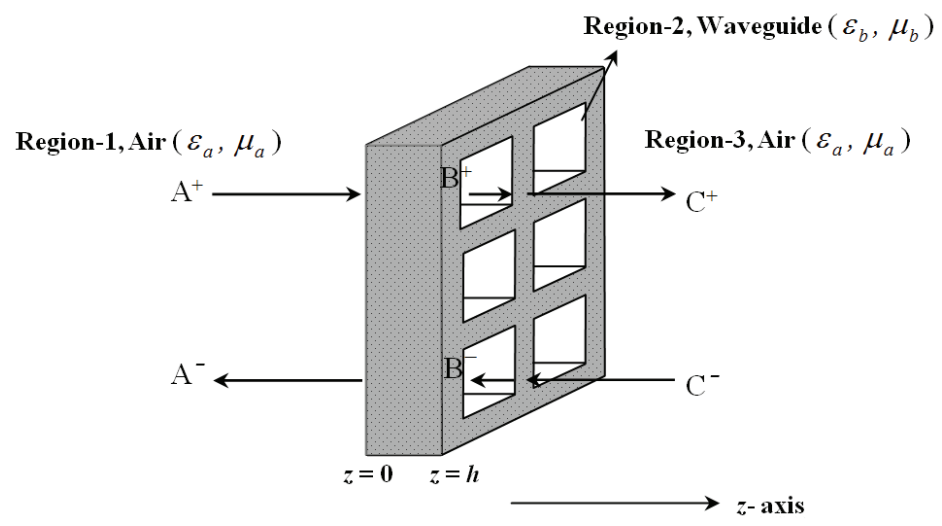

Figure 2. Side view of thick FSS perforated by rectangular apertures. 
3 , respectively. The scattering matrix at the interface $z=0$ is given as

$$
\left(\begin{array}{c}
A_{0}^{-} \\
B_{0}^{+}
\end{array}\right)=\left(\begin{array}{ll}
S_{11}^{a} & S_{12}^{a} \\
S_{21}^{a} & S_{22}^{a}
\end{array}\right)\left(\begin{array}{c}
A_{0}^{+} \\
B_{0}^{-}
\end{array}\right)
$$

where the scattering parameters $S^{a}$ are given by ${ }^{9}$.

The scattering matrix at the interface $z=h$ is given as

$$
\left(\begin{array}{c}
B_{h}^{-} \\
C_{h}^{+}
\end{array}\right)=\left(\begin{array}{ll}
S_{11}^{b} & S_{12}^{b} \\
S_{21}^{b} & S_{22}^{b}
\end{array}\right)\left(\begin{array}{l}
B_{h}^{+} \\
C_{h}^{-}
\end{array}\right)
$$

Since the screen is perfectly conducting and aperture region is filled with air, the EM propagation through the screen will be lossless. The junction at $z=h$ is the mirror image of the junction at $z=0$. The scattering matrix at the interface $z=h$ will be transpose to the matrix that of at $z=0$ interface, which is given by

$$
\left.\begin{array}{l}
S_{11}^{b}=S_{22}^{a} \\
S_{12}^{b}=S_{21}^{a} \\
S_{21}^{b}=S_{12}^{a} \\
S_{22}^{b}=S_{11}^{a}
\end{array}\right\}
$$

Now, the scattering matrices $S^{a}$ and $S^{b}$ are cascaded using propagation matrix $P$ for the aperture region, to obtain the final scattering matrix $S^{c}$ of the complete FSS structure. The scattering parameters are given by

$$
\left.\begin{array}{l}
S_{11}^{c}=S_{11}^{a}+S_{12}^{a} P\left(I-P S_{11}^{b} P S_{22}^{a}\right)^{-1} S_{11}^{b} P S_{21}^{a} \\
S_{12}^{c}=S_{12}^{a} P\left(I-P S_{11}^{b} P S_{22}^{a}\right)^{-1} S_{12}^{b} \\
S_{21}^{c}=S_{21}^{b} P\left(I-S_{22}^{a} P S_{11}^{b} P\right)^{-1} S_{21}^{a} \\
S_{22}^{c}=S_{22}^{b}+S_{21}^{b} P\left(I-S_{22}^{a} P S_{11}^{b} P\right)^{-1} S_{22}^{a} P S_{12}^{b}
\end{array}\right\}
$$

where $P=e^{-j \gamma_{m n} h}$ is given by ${ }^{9}$. The above expressions represent the components of generalized scattering matrix of the proposed FSS structure and are used to determine its transmission and reflection characteristics.

\section{DISCUSSION OF RESULTS}

In this work, a copper sheet of thickness $0.11 \mathrm{~cm}$ perforated by an array of rectangular apertures has been considered for the EM analysis. The dimensions of rectangular apertures are: $a$ $=1.80 \mathrm{~cm}$ wide (along X-axis); and $b=0.55 \mathrm{~cm}$ high (along $\mathrm{Y}$-axis). The periodicity of the array along $\mathrm{X}$ - and $\mathrm{Y}$ - directions is: $\mathrm{T}_{\mathrm{x}}=2.325 \mathrm{~cm}$ and $\mathrm{T}_{\mathrm{y}}=1.555 \mathrm{~cm}$ respectively as shown in Fig. 1. Here rectangular lattices are considered, i.e., $\alpha=90^{\circ}$ inside the FSS structure. Here, the Floquet modes with index 10 i.e., $10<m, n>-10$ and waveguide mode with index 9 i.e., $p, q \leq 9$ have been used in the computation. The variation of transmission characteristics with frequency at $\theta=0^{\circ}$ and $30^{\circ}$ for TM incidence wave is shown in Figs. 3 and 4, respectively, where the computed results are compared with those reported (theoretical and experimental) results in the open literature ${ }^{8}$. It is observed that the computed results are in excellent agreement with the reported results, which are based on the

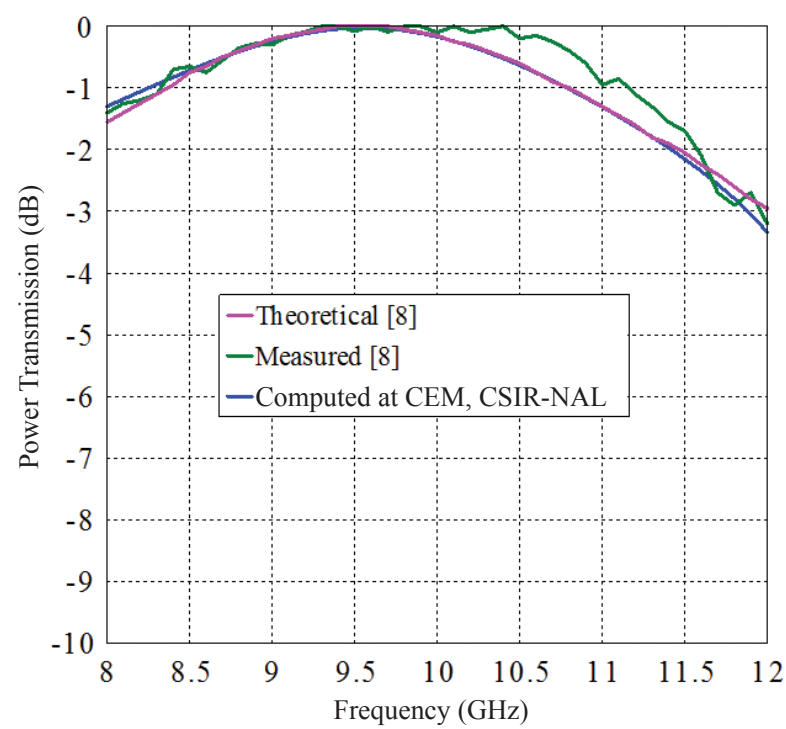

Figure 3. Transmission characteristics of a thick FSS for TM incidence wave at $\theta=0^{\circ}$

mode matching (MM)-finite element method (FEM) method.

The computation time taken by 32-bit operating system on Intel Core (TM) 2 Duo CPU and 4 GB RAM is around 4.38 second. Further, the transmission characteristics of thick FSS perforated by rectangular apertures are studied at $\theta=45^{\circ}, 60^{\circ}$, and $80^{\circ}$ for $\mathrm{TM}$ incidence wave as shown in Fig. 5. The transmission characteristics of the proposed FSS structure at normal incidence for different dielectric materials inside the aperture (waveguide) region are shown in Fig. 6, where the dielectric loss of the material is not considered in the computation. It is observed that the resonance frequency of the transmission characteristics decreases and curves become steeper as dielectric constant of the material increases. The dependence of transmission characteristics of a thick FSS structure for different loss tangents (keeping $\varepsilon_{\mathrm{r}}=4.0$ ) of materials inside the aperture at normal incidence is shown in Fig. 7. It is found that transmission reduces with the increase in the loss tangents of the dielectric material.

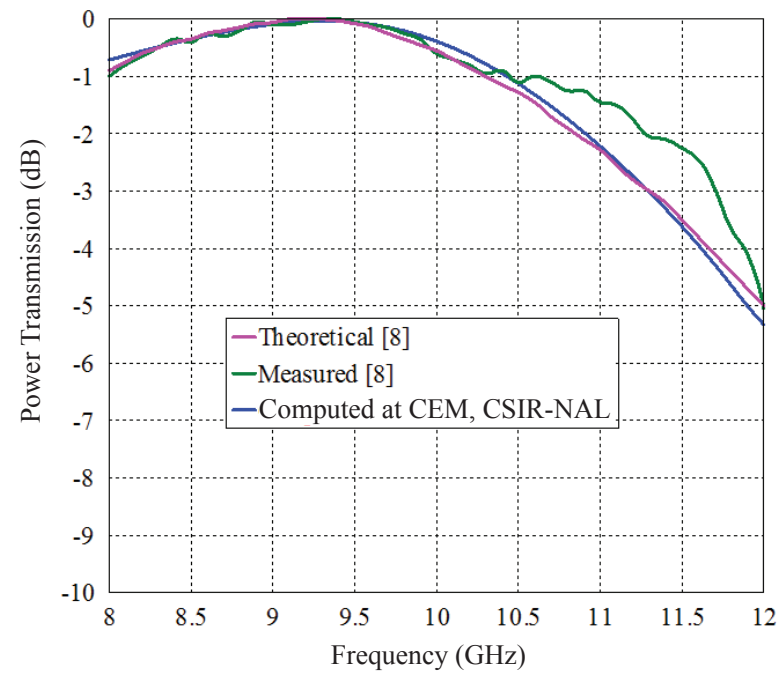

Figure 4. Transmission characteristics of a thick FSS for TM incidence wave at $\theta=30^{\circ}$. 


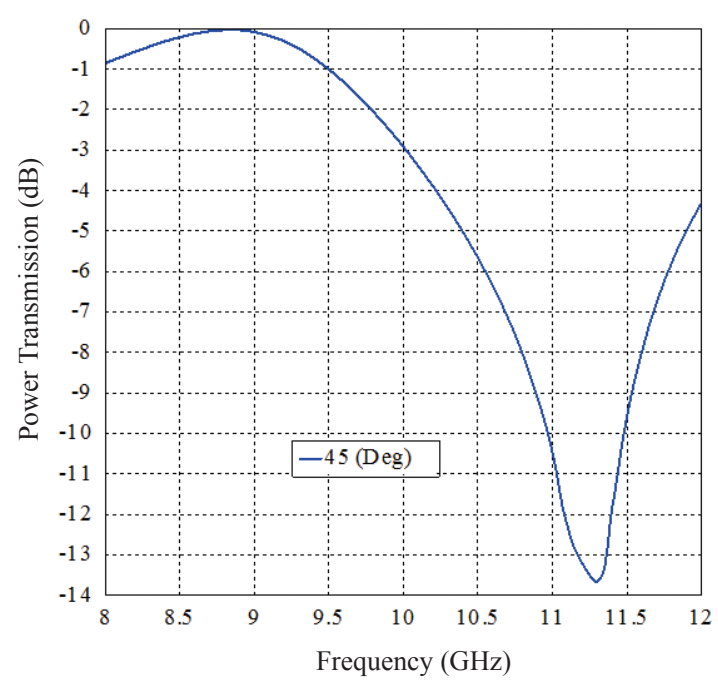

(a)

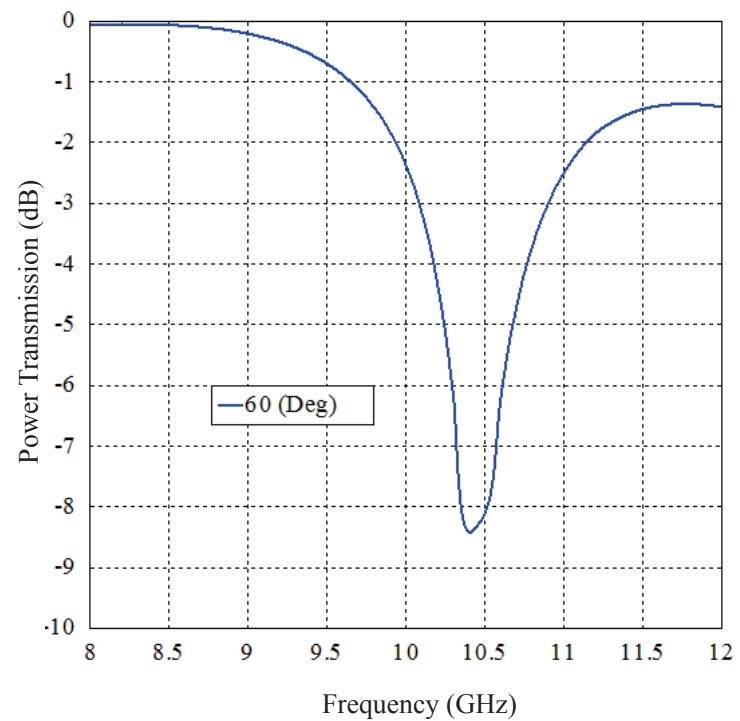

(b)

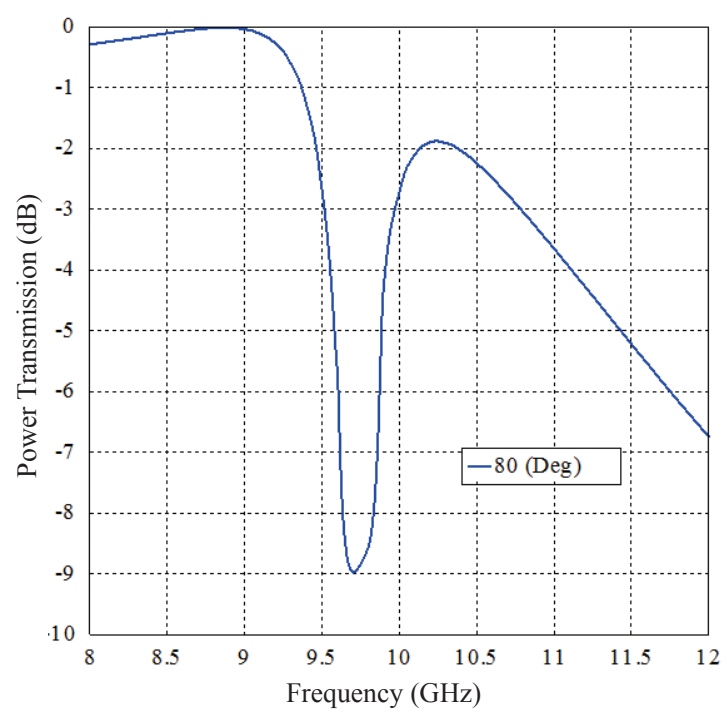

(c)

Figure 5. Transmission characteristics of a thick FSS for TM incidence wave at angle of incidence: (a) $\theta=45^{\circ}$, (b) $\theta=\mathbf{6 0}^{\circ}$, (c) $\theta=\mathbf{8 0}^{\circ}$.

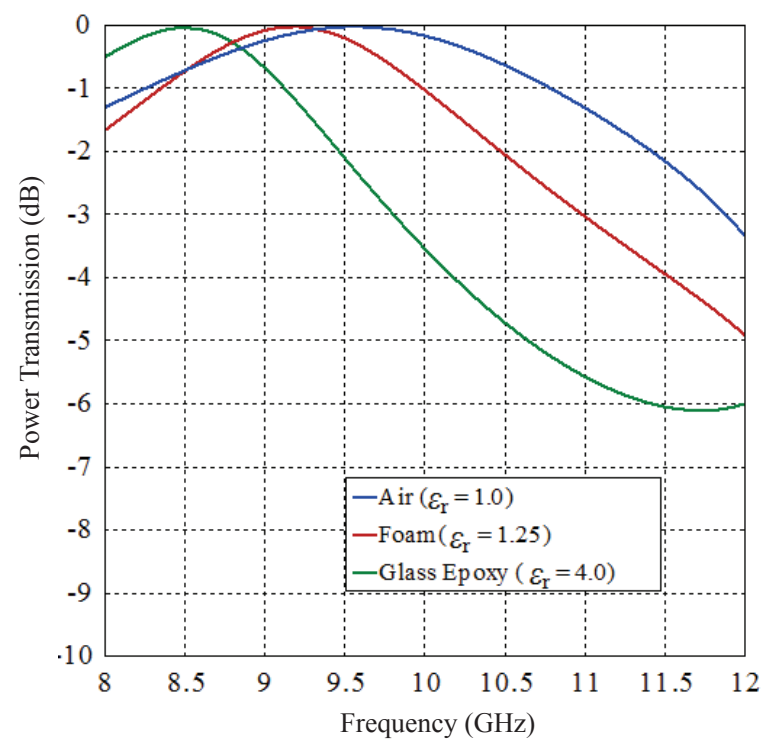

Figure 6. Transmission characteristics for different lossless dielectric materials inside the aperture region at normal incidence.

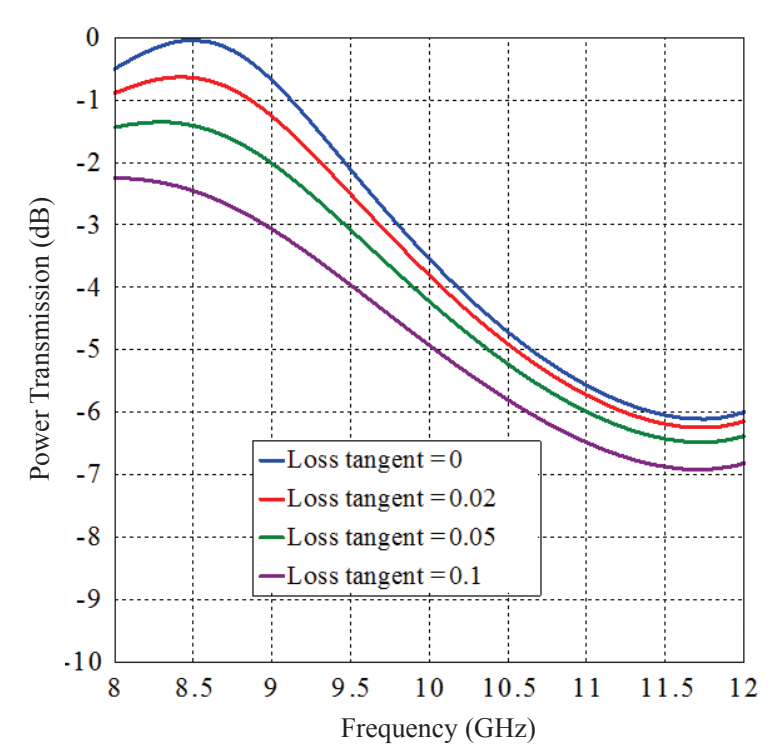

Figure 7. Transmission characteristics for different loss tangents of material (keeping $\varepsilon_{\mathrm{r}}=4.0$ ) inside the aperture at normal incidence.

\section{CONCLUSIONS}

The analysis of thick FSS perforated by rectangular apertures has been carried out in this paper using MM-GSM technique for TM plane wave incidence. The computed results are compared with the reported results at incidence angle $\theta=0^{\circ}$ and $30^{\circ}$ and found to be in excellent agreement with the reported results. For streamlined applications, the transmission characteristics are also studied at higher angles of incidence $\left(\theta=45^{\circ}, 60^{\circ}\right.$, and $\left.80^{\circ}\right)$. Finally, the dependence of transmission on the dielectric parameters of the material inside the aperture region is studied at normal incidence. The proposed thick FSS structure is found to be a better choice for the design of airborne radomes. 


\section{REFERENCES}

1. $\mathrm{Wu}$, T.K. Frequency selective surface and grid array. John Wiley \& Sons, New York, 1995, pp. 331.

2. Imbriale, W.A. Analysis of a thick dichroic plate with arbitrary shaped holes. Jet Propulsion Laboratory, Pasadena, CA, IPN Progress Report: 2001, pp. 42-146.

3. Dubrovka, R.; Vazquez, J.; Parini, C. \& Moore, D. Equivalent circuit method for analysis and synthesis of frequency-selective surfaces. IEE Proc. H Microwaves, Ant. Propag., 2006, 153(3), 213-220.

4. A Bhattacharyya, A.K. On the convergence of MoM and mode matching solutions for infinite array and waveguide problems. IEEE Tran. Ant. Propag., 2003, 51(7), 15991606.

5. Zheng, L. \& Jin, J.M. Analysis of 3D frequency selective structures using a high-order finite element method. Microwave Optical Technol. Let., 2003, 38(4), 259-263.

6. Campos, A.L. P.S. Transverse transmission line method to analyze stop-band microwave filter. Microwave Optical Technol. Let., 2007, 49(11), 2678-2681.

7. Loui, H. Modal analysis and design of compound gratings and frequency selective surfaces. Department of Electrical and Computer Engineering, University of Colorado, Boulder, 2006, PhD Thesis.

8. Widenberg, B.; Poulsen, S. \& Karlsson, A. Thick screens perforated with a periodic array of aperture with arbitrary cross-section. Department of Electroscience, Lund Institute of Technology, Sweden, Electromagnetic Theory Tech Report No. TEAT-7082, June 2001.

9. Narayan, S.; Nair, R.U. \& Jha, R.M. EM performance analysis of thick rectangular aperture-type FSS based on MM-GSM technique. In the Proceedings of the APSYM 2010, Antennas and Propagation Symposium, Kochi, India, December 14-16, 2010, pp. 235-238.

\section{Contributors}

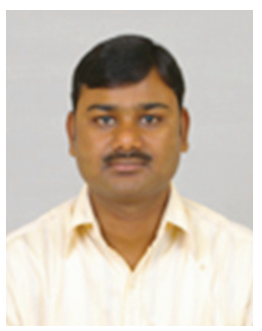

Dr Shiv Narayan received his $\mathrm{PhD}$ (Electronics Eng.) from Indian Institute of Technology (Banaras Hindu University), Varanasi, India in 2006. He is currently working as a Scientist at Centre for Electromagnetics (CEM) of CSIR-National Aerospace Laboratories (NAL), Bangalore, India. He has published 35 technical documents including journal and conferences papers. His research interests are broadly in the field of electromagnetics applications and the topics include: Frequency selective surfaces, metamaterials, numerical methods in electromagnetics, EM material characterization, microstrip patch antenna, and pattern synthesis of antenna array.

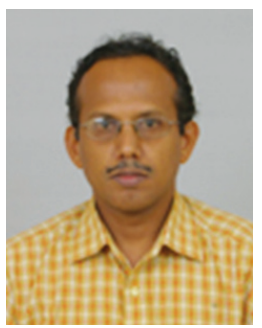

Dr Raveendranath $\mathbf{U}$. Nair received his MSc and PhD in Physics (Microwave Electronics) Mahatma Gandhi University, Kerala, India, in 1989 and 1997, respectively. Presently he is working as Scientist at the CEM CSIR-NAL, Bangalore, India where currently he is a principal scientist. He was the recipient of CSIR-NAL Excellence in Research Award for the year 2007-2008. He has authored/co-authored over 100 research publications. His research interests include: Computational electromagnetics, EM design, analysis and performance measurements of radomes, metamaterials, frequency selective surfaces, EM material characterization techniques.

Dr Rakesh Mohan Jha obtained his PhD (Engg.) Indian

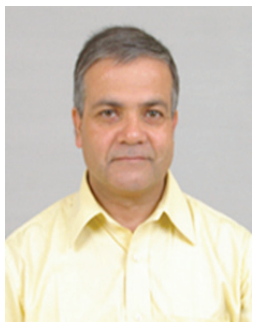
Institute of Science, Bangalore, in 1989. He is currently a Chief Scientist and Head of Centre for Electromagnetics (CEM) at CSIR-NAL, Bangalore. He has published more than 350 scientific research papers and technical reports. He was awarded Sir CV Raman Award for Aerospace Engineering for the Year 1999. His active areas of research are in the domain of computational electromagnetics (CEM) for aerospace applications; these include GTD/UTD, 3-D ray tracing and surface modelling, aerospace antennas and radomes, radar cross section studies, radar absorbing materials and radar absorbing structures, and metamaterials for aerospace applications. 